\title{
Defining Ischemic Core in Acute Ischemic Stroke Using CT Perfusion: A Multiparametric Bayesian-Based Model
}

(D) K. Nael, (D) E. Tadayon, (DD. Wheelwright, (D)A. Metry, (D).T. Fif, DS. Tuhrim, (DR.A. De Leacy, (D)A.H. Doshi, (D) H.L. Chang, and (i). Mocco

\begin{abstract}
BACKGROUND AND PURPOSE: The Bayesian probabilistic method has shown promising results to offset noise-related variability in perfusion analysis. Using CTP, we aimed to find optimal Bayesian-estimated thresholds based on multiparametric voxel-level models to estimate the ischemic core in patients with acute ischemic stroke.
\end{abstract}

MATERIALS AND METHODS: Patients with anterior circulation acute ischemic stroke who had baseline CTP and achieved successful recanalization were included. In a subset of patients, multiparametric voxel-based models were constructed between Bayesian-processed CTP maps and follow-up MRIs to identify pretreatment CTP parameters that were predictive of infarction using robust logistic regression. Subsequently CTP-estimated ischemic core volumes from our Bayesian model were compared against routine clinical practice oscillation singular value decomposition-relative cerebral blood flow $<30 \%$, and the volumetric accuracy was assessed against final infarct volume.

RESULTS: In the constructed multivariate voxel-based model, 4 variables were identified as independent predictors of infarction: TTP, relative CBF, differential arterial tissue delay, and differential mean transit time. At an optimal cutoff point of 0.109 , this model identified infarcted voxels with nearly $80 \%$ accuracy. The limits of agreement between CTP-estimated ischemic core and final infarct volume ranged from -25 to $27 \mathrm{~mL}$ for the Bayesian model, compared with -61 to $52 \mathrm{~mL}$ for oscillation singular value decomposition-relative CBF.

CONCLUSIONS: We established thresholds for the Bayesian model to estimate the ischemic core. The described multiparametric Bayesian-based model improved consistency in CTP estimation of the ischemic core compared with the methodology used in current clinical routine.

ABBREVIATIONS: AIS = acute ischemic stroke; ATD = arterial tissue delay; AUC = area under the curve; diff = differential; oSVD = oscillation singular value decomposition; $\mathrm{rCBF}=$ relative $\mathrm{CBF}$

n patients with acute ischemic stroke (AIS), characterization of ischemic core on baseline imaging is essential for proper treatment decision-making. ${ }^{1-3}$ While DWI provides the most accurate estimation of ischemic core, ${ }^{4,5}$ CTP can improve the diagnostic yields of CT-based imaging techniques for delineation of ischemic core to approach those of MR imaging. ${ }^{6-8}$

Following successful implementation of CTP for improved

Received March 26, 2019; accepted after revision July 7.

From the Department of Radiology (K.N., E.T., A.M., A.H.D.), Neuroimaging Advanced and Exploratory Lab, and Departments of Neurology (D.W., J.F., S.T.), Neurosurgery (J.F., R.A.D.L., J.M.), and Population Health Science and Policy (H.C.), Icahn School of Medicine at Mount Sinai, New York, New York.

Preliminary results of this article previously presented at: International Stroke Conference, February 5-8, 2019; Honolulu, Hawaii. Abstract: WP87.

Please address correspondence to Kambiz Nael, MD, Icahn School of Medicine at Mount Sinai, Department of Radiology, One Gustave L. Levy Place, Box 1234, New York, New York; e-mail: Kambiznael@gmail.com; @kambiznael

-- Indicates open access to non-subscribers at www.ajnr.org

http://dx.doi.org/10.3174/ajnr.A6170 treatment selection in patients presenting with large-vessel occlusion and up to 24 hours from the onset, ${ }^{9,10} \mathrm{CTP}$ is now included in the latest American Heart Association guidelines for treatment selection in patients with anterior circulation large-vessel occlusion who present beyond 6 hours from the onset of symptoms (class I, level of evidence A). ${ }^{11}$

As quantitative CTP is gaining momentum for widespread clinical use, neurology and radiology communities need to be aware of its potential pitfalls. One of the disadvantages of CTP is substantial variability and the potential for erroneous estimation of the ischemic core, which may be at least partially related to the inherently noisy nature of CTP datasets. ${ }^{12,13}$ Because an ischemic core volume of $>50-70 \mathrm{~mL}^{14,15}$ could potentially exclude patients from a life-saving treatment, this pitfall could have important therapeutic and prognostic implications.

Although recent application of quantitative CTP has shown promising results in the supervised and controlled environment of clinical trials, there remains variability and inconsistency in the 
accuracy of quantitative CTP data provided in routine daily practice. $^{13,16-20}$

The Bayesian method is a robust probabilistic method that minimizes the effects of oscillation and high levels of noise during residue function estimation compared with other deconvolution methods. ${ }^{21,22}$ The advantages of the Bayesian model to provide more accurate estimation of perfusion values and reduction of variability have been shown in experimental phantom studies ${ }^{23,24}$ and also recently in a cohort of patients with AIS. ${ }^{25}$

In this study we had 2 aims: first, to define the optimal Bayesian-based thresholds for estimation of ischemic core. In particular, we aimed to develop a multiparametric model from our CTP datasets that provides a high degree of accuracy in the estimation of ischemic core in comparison with MR imaging. The second aim was a comparative analysis between the diagnostic accuracy of our Bayesian-based model and routinely used postprocessing methodology to estimate the ischemic core from CTP datasets in patients with AIS.

\section{MATERIALS AND METHODS \\ Patients}

We included consecutive patients with AIS who presented to our institution between January 2016 and July 2018 and had the following inclusion criteria: 1) anterior circulation ischemic stroke with proximal arterial occlusion (carotid or MCA); 2) baseline CTP; 3 ) successful recanalization defined by TICI $\geq 2$ b via mechanical thrombectomy; and 4) follow-up imaging within 1 week after recanalization to calculate the final infarct volume. We excluded patients who had the following: 1) time from CTP to recanalization of $>4$ hours; 2 ) rethrombosis/reocclusion of the recanalized artery on follow-up MRA/CTA; or 3) development of significant intracranial hemorrhage that precluded accurate estimation of infarct volume on follow-up imaging. The study was performed under an approved institutional review board guideline.

\section{CTP Image Acquisition}

CTP was performed on 2 CT scanners, a LightSpeed VCT (GE Healthcare, Milwaukee, Wisconsin) and a Somatom Definition (Siemens, Erlangen, Germany). We used the following scan parameters: $100-\mathrm{mm}$ coverage in the z-axis, $80 \mathrm{kV}, 150 \mathrm{~mA}$, effective dose $=3.3 \mathrm{mSv}$, slice thickness $=5 \mathrm{~mm}$, collimation $=64 \times 0.625 \mathrm{~mm}$. Total acquisition time was 60 seconds ( 30 consecutive spiral acquisitions of 2 seconds each). A total of $50 \mathrm{~mL}$ of Isovue-370 (iopamidol; Bracco, Princeton, New Jersey) was injected intravenously followed by a $20-\mathrm{mL}$ saline flush at $5 \mathrm{~mL} / \mathrm{s}$.

\section{Image Analysis}

CTP data were processed using FDA-approved postprocessing software (Olea Sphere Version 6.0; Olea Medical, La Ciotat, France). First, the arterial input function was detected automatically using a cluster-analysis algorithm. ${ }^{26}$ This arterial input function was subsequently used by the Bayesian probabilistic method $^{22}$ to generate the perfusion parametric maps, including TTP, CBF, CBV, arterial tissue delay (ATD), and MTT. Relative values for CBF and CBV (rCBF, relative CBV) were calculated by dividing the absolute values of each voxel within the infarction mask by the mean value of the contralateral side.

Model Development (Voxel-Based Analysis). The first 19 patients who had pretreatment CTP and postthrombectomy MR imaging were included for model development. For image registration and analysis, the CTP template was first thresholded to $0-180 \mathrm{HU}$ and the brain was extracted from the CTP template using the FSL Brain Extraction Tool $(f=0.01)$ (BET; https://fsl.fmrib.ox.ac.uk/ $\mathrm{fs} /$ /fslwiki/BET). To account for regional differences in perfusion values across brain areas, 5 additional maps were generated (difference maps) by subtracting the mean value of a cube of 27 voxels centered on the voxel exactly on the contralateral hemisphere of each voxel. The contralateral voxel was defined as the voxel on the opposite hemisphere that had a similar Euclidean distance from the center (anterior commissure) on the axial plane.

These differential (diff) maps $\left(\mathrm{TTP}_{\text {diff, }}, \mathrm{rCBF}_{\text {diff, }}\right.$, relative $\mathrm{CBV}_{\text {diff, }}, \mathrm{ATD}_{\text {diff, }}$, and $\left.\mathrm{MTT}_{\text {diff }}\right)$ in addition to the 5 primary parametric maps (a total of 10 variables) were included in imageanalysis and predictive modeling. Extracted brain from all CTP maps was linearly transformed and registered to brain MR imaging using the FMRIB Linear Image Registration Tool (FLIRT; https://fsl.fmrib.ox.ac.uk/fsl/fslwiki/FLIRT) with $9 d f$ and a mutual information cost function. All registrations were visually inspected for accuracy.

An infarction mask and 2 noninfarction masks, 1 on the ipsilateral and 1 on the contralateral side of the lesion, were drawn on the MR imaging for each subject by a board-certified neuroradiologist. Coregistered DWI was available to guide and confine the VOI to the region of acute infarction. All CTP voxel values from these 3 masks in each patient were exported for statistical analysis.

Volumetric Analysis. The final output of our Bayesian-based predictive model defined by its logit scores (see Results) was used to calculate the ischemic core volume in the remaining patients $(n=$ 48 ), whose CTP data were not used for model development. The logit scores were programmed into a software developmental kit (Olea Infinite Software Developmental Kit; Olea Medical). The DICOM images of Bayesian-processed CTP maps were exported into this software to calculate the ischemic core volume using the logit scores of our model.

In addition, the CTP-estimated ischemic core volume for each patient was calculated by applying the broadly used threshold methodology $(\mathrm{rCBF}<30 \%)^{7}$ currently applied in clinical practice using an oscillation singular value decomposition (oSVD). ${ }^{27}$

The volume of final infarction was calculated by applying a volume of interest on the DWI hyperintense region using a voxel-based signal intensity method subsuming the entire region of DWI hyperintensity. When follow-up MR imaging was not available, follow-up CT within 24-48 hours from thrombectomy was used for determination of final infarct volume. In these patients, the infarct (defined as established hypodense regions) was manually delineated by a neuroradiologist with 10 years of experience. 


\section{Statistical Analysis}

Voxel-level logistic regression models were used to identify pretreatment CTP parameters (5 parametric maps +5 differential maps) that were predictive of infarction. Voxels from baseline CTP that fell within the MR imaging-defined infarct area were considered true infarction, and all other voxels were considered not infarcted. Because the infarct status among voxels from the same subject may be correlated, the Huber-White standard errors (ie, robust standard errors) were computed to account for the additional source of variability. CTP parameters that were significant at the .15 level in the univariate logistic regression analysis were considered for the multivariable model. A backward-selec-

Table 1: Voxel-based univariate analysis of infarction and noninfarction voxels ${ }^{\mathrm{a}}$

\begin{tabular}{lccc}
\hline Variable & Infarction & Noninfarction & $\boldsymbol{P V a l u e}^{\mathbf{b}}$ \\
\hline TTP & $32.00(1.95)$ & $24.30(1.19)$ & $<.001$ \\
rCBF & $24.78(2.81)$ & $48.43(6.49)$ & $<.001$ \\
rCBV & $2.81(0.46)$ & $4.09(0.56)$ & .02 \\
ATD & $3.58(0.18)$ & $1.49(0.16)$ & $<.001$ \\
MTT & $6.58(0.58)$ & $5.33(0.15)$ & .01 \\
rCBF $_{\text {diff }}$ & $-25.60(10.93)$ & $3.20(1.20)$ & .02 \\
rCBV $_{\text {diff }}$ & $-1.15(0.52)$ & $0.06(0.06)$ & .03 \\
TTP diff $_{\text {ATD }}$ & $8.39(0.41)$ & $0.25(0.17)$ & $<.001$ \\
ATD $_{\text {diff }}$ & $2.15(0.17)$ & $-0.16(0.06)$ & $<.001$ \\
MTT $_{\text {diff }}$ & $2.19(0.49)$ & $-0.12(0.06)$ & $<.001$ \\
\hline
\end{tabular}

Note:- $r C B V$ indicates relative CBV.

${ }^{a}$ The values are presented as mean (SD). The SDs reported are the Huber-White (robust) standard errors. All units are in seconds, except for rCBF and rCBV, which are unitless.

${ }^{b} P$ values are based on a linear regression model with the presence of infarct as the independent variable and imaging parameters as the outcome.

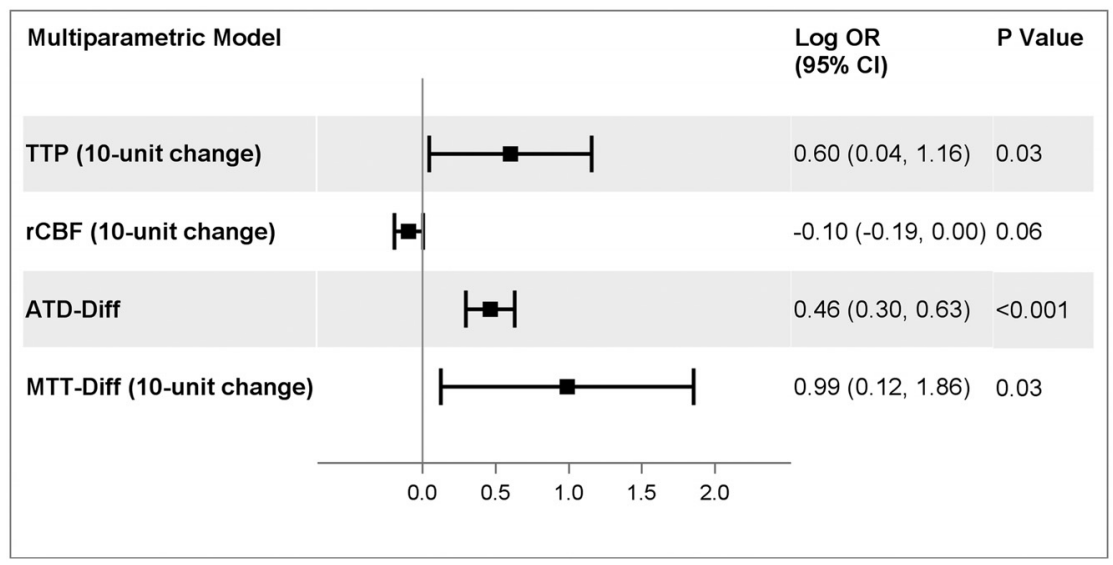

FIG 1. Multiparametric voxel-based model for infarction.

Table 2: Optimal threshold, sensitivity, specificity, and accuracy for TTP, rCBF, ATD diff, MTT $_{\text {diff, }}$, and the final model in identifying infarcted voxels

\begin{tabular}{lccccc}
\hline \multicolumn{1}{c}{ Variable } & Threshold & Sensitivity & Specificity & Accuracy & AUC \\
\hline TTP & 28.82 seconds & $65.3 \%$ & $77.9 \%$ & $76.5 \%$ & 0.76 \\
rCBF & 22.10 & $60.0 \%$ & $72.9 \%$ & $71.5 \%$ & 0.73 \\
ATD $_{\text {diff }}$ & 0.87 seconds & $68.1 \%$ & $80.2 \%$ & $78.9 \%$ & 0.80 \\
MTT $_{\text {diff }}$ & 1.38 seconds & $56.2 \%$ & $74.5 \%$ & $72.5 \%$ & 0.69 \\
Final model $^{\mathrm{a}}$ & $0.109^{\mathrm{b}}$ & $74.2 \%$ & $80.0 \%$ & $79.4 \%$ & 0.84 \\
\hline
\end{tabular}

a The final model consisted of TTP, $\mathrm{rCBF}, \mathrm{ATD}_{\text {diff, }}$ and $\mathrm{MTT}_{\text {diff }}$ as the independent variables and the presence of infarct as the outcome.

${ }^{b}$ Equation of the final model: logit $(P)=-3.9170+0.0601 \times \mathrm{TTP}-0.0095 \times \mathrm{rCBF}+0.4629 \times \mathrm{ATD}_{\text {diff }}+0.0989 \times$ $\mathrm{MTT}_{\text {diff }}$ where logit $(P)=$ estimated log odds of infarction for a given voxel. If logit $(P)$ is greater than the optimal threshold of 0.109 , the voxel is classified as infarct. tion approach was then used to identify significant variables at the .05 level.

The final multiparametric model was assessed using receiver operating characteristic curve analysis. The optimal cutoff point (measured on the logit scale) that identified a voxel as infarct was determined by the Youden index. Summary measures such as sensitivity, specificity, and accuracy were calculated on the basis of the optimal threshold to quantify how well the final model differentiated between infarct and noninfarct voxels.

Finally, the CTP-estimated ischemic core volumes obtained from oSVD-rCBF $<30 \%$ (that is used in routine practice) and from the multiparametric Bayesian model (optimal logit score) were compared against the final infarct volume using Bland-Altman methods. The optimal volumetric agreement was determined by the smallest mean magnitude (absolute) difference in lesion volume. All statistical analyses were performed at the .05 (2-sided) significance level using SAS 9.4 (SAS Institute, Cary, North Carolina), R 3.1.1 statistical and computing software (http://www.r-project.org), and SPSS 17.0 (IBM, Armonk, New York).

\section{RESULTS}

A total of 88 charts were reviewed with 67 patients ( 40 women, 27 men) available for analysis. Two patients were excluded due to development of a large intracranial hemorrhage. Two patients were excluded because they had rethrombosis of the recanalized MCA on follow-up MRA. Nine patients were excluded because of nondiagnostic CTP (significant motion, $n=4$ ), insufficient contrast bolus $(n=2)$, and a truncated arterial input function $(n=$ 3). Eight patients were excluded due to having $>4$ hours of recanalization time from the onset of CTP. The mean age was $69.6 \pm 14.4$ years. The median stroke severity measured by the NIHSS was 15 (interquartile range, 9-21). A total of $15(22 \%)$ patients had internal carotid artery occlusion, and $52(78 \%)$ had proximal MCA occlusion (M1, $n=38$; M2, $n=14)$. The mean of time from symptom onset was $8.7 \pm 5.2$ hours. A total of 25 patients presented $<6$ hours from the onset, and 42 presented after 6 hours. The median time from CTP to recanalization was 1.8 hours (interquartile range, 1.3-2.7 hours), and from CTP to follow-up imaging used for determination of final infarction, it was 24 hours (interquartile range, 19-31 hours). Final recanalization scores were TICI $2 \mathrm{~b}$ $(n=22,33 \%)$, TICI $2 \mathrm{c}(n=16,24 \%)$ and TICI $3(n=29,43 \%)$.

\section{Voxel-Based Analysis}

A total of 2,577,261 voxels in 19 patients were included in the voxel-based analysis. All 10 imaging variables were significantly associated with infarction in the univariate analysis (Table 1). The final 


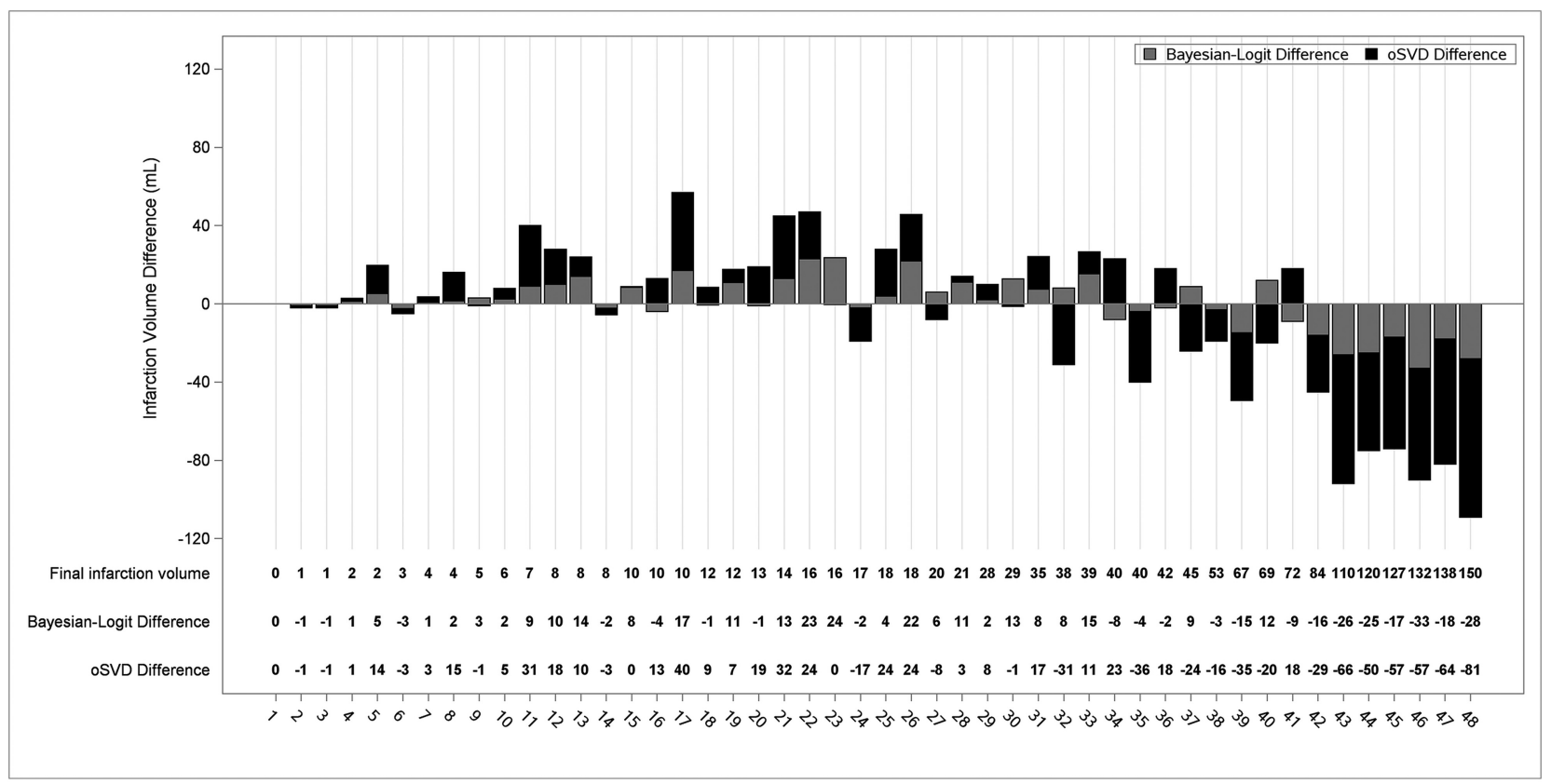

FIG 2. Stacked bar graph visualization of errors in ischemic core volume estimation compared with final infarction volume by Bayesian-logit and OSVD postprocessing. The error values are noticeably greater with oSVD (black bars) compared with Bayesian-logit (gray bars).

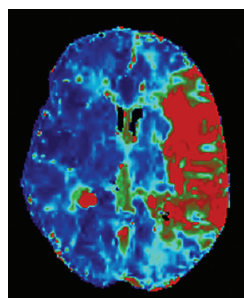

TTP

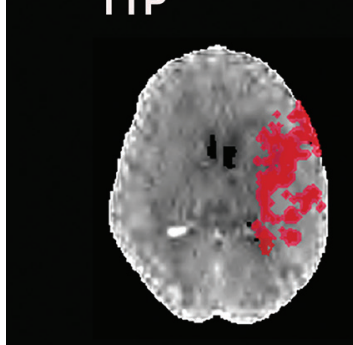

OSVD ( $\mathrm{rCBF}<30 \%)$

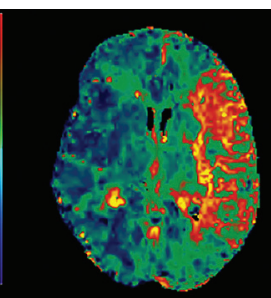

MTT

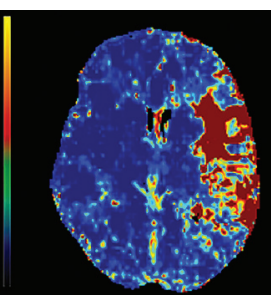

ATD

FIG 3. A 74-year-old woman who presented with left Ml occlusion and a baseline NIHSS score of 26. She underwent successful mechanical thrombectomy $(\mathrm{TICI} 2 \mathrm{C})$. The time from CTP to recanalization was 78 minutes, and the time from CTP to MR imaging was 19 hours. The 4 CTP maps included in our final model are shown. Note that the estimated ischemic core derived from our multiparametric Bayesian-logit model provides more accurate estimation of final infarction on MR imaging in comparison with what is estimated from oSVD-CBF $<30 \%$ (current clinical practice).

multiparametric model contained 4 variables that remained independent predictors of infarction when evaluated against other imaging parameters. The CTP measures that were associated with an increased log odds of infarction included TTP (10-unit change, log odds ratio, $0.60 ; 95 \% \mathrm{CI}, 0.04-1.16 ; P=.03)$, $\operatorname{ATD}_{\text {diff }}(\log$ odds ratio, 0.46 ; $95 \% \mathrm{CI}, 0.30-0.63 ; P<.001)$, and $\mathrm{MTT}_{\text {diff }}(10-$ unit change, $\log$ odds ratio, $0.99 ; 95 \% \mathrm{CI}, 0.12-1.86 ; P=.03$ ). Higher rCBF was protective (10-unit change, log odds ratio, -0.10 ; $95 \% \mathrm{CI},-0.19$ to $0 ; P=.058$ ) (Fig 1 ). The parameters

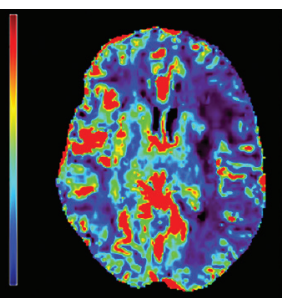

CBF relative CBV and MTT were highly correlated with $\mathrm{rCBF}$ and $\mathrm{MTT}_{\text {diff, }}$ respectively, and were subsequently removed.

Based on the final model, the optimal cut-point value (ie, optimal logit score) that discriminated infarcted voxels from noninfarcted ones was 0.109. At this threshold, the accuracy of the classification was $79.4 \%$, with a sensitivity of $74.2 \%$ and a specificity of $80 \%$. The area under the receiver operating characteristic curve (AUC) was 0.84 (Table 2). Receiver operating characteristic analysis was also performed on the individual components of the final model. The optimal thresholds identified were: TTP, 28.82 seconds $(\mathrm{AUC}=0.76) ; \mathrm{rCBF}, 22.1$ $(\mathrm{AUC}=0.73) ; \mathrm{ATD}_{\mathrm{diff}}, 0.87$ second $(\mathrm{AUC}=0.80)$; and $\mathrm{MTT}_{\text {diff }}, 1.38 \mathrm{sec}-$ onds (AUC $=0.69)$.

\section{Volumetric Analysis}

Volumetric analysis was based on 48 patients whose CTP data were not used to develop the voxel-level models. The mean final infarct volume calculated from the follow-up MR imaging $(n=40)$ and CT $(n=8)$ was $36 \mathrm{~mL}( \pm 41)$. The mean estimated ischemic core volumes were $31 \mathrm{~mL}( \pm 24)$ for oSVD and $37 \mathrm{~mL}( \pm 33)$ for the Bayesian-based logit score.

The means of differences between CTP-estimated ischemic core volume and final infarct volume were $-4 \mathrm{~mL}$ (95\% CI, -13 to $4 \mathrm{~mL}$ ) for oSVD-CBF $<30 \%$ and $1 \mathrm{~mL}(95 \% \mathrm{CI},-3$ to $5 \mathrm{~mL}$ ) for the Bayesian-based logit score. The individualized error bars for estimation of the final infarct volume between the Bayesian- 


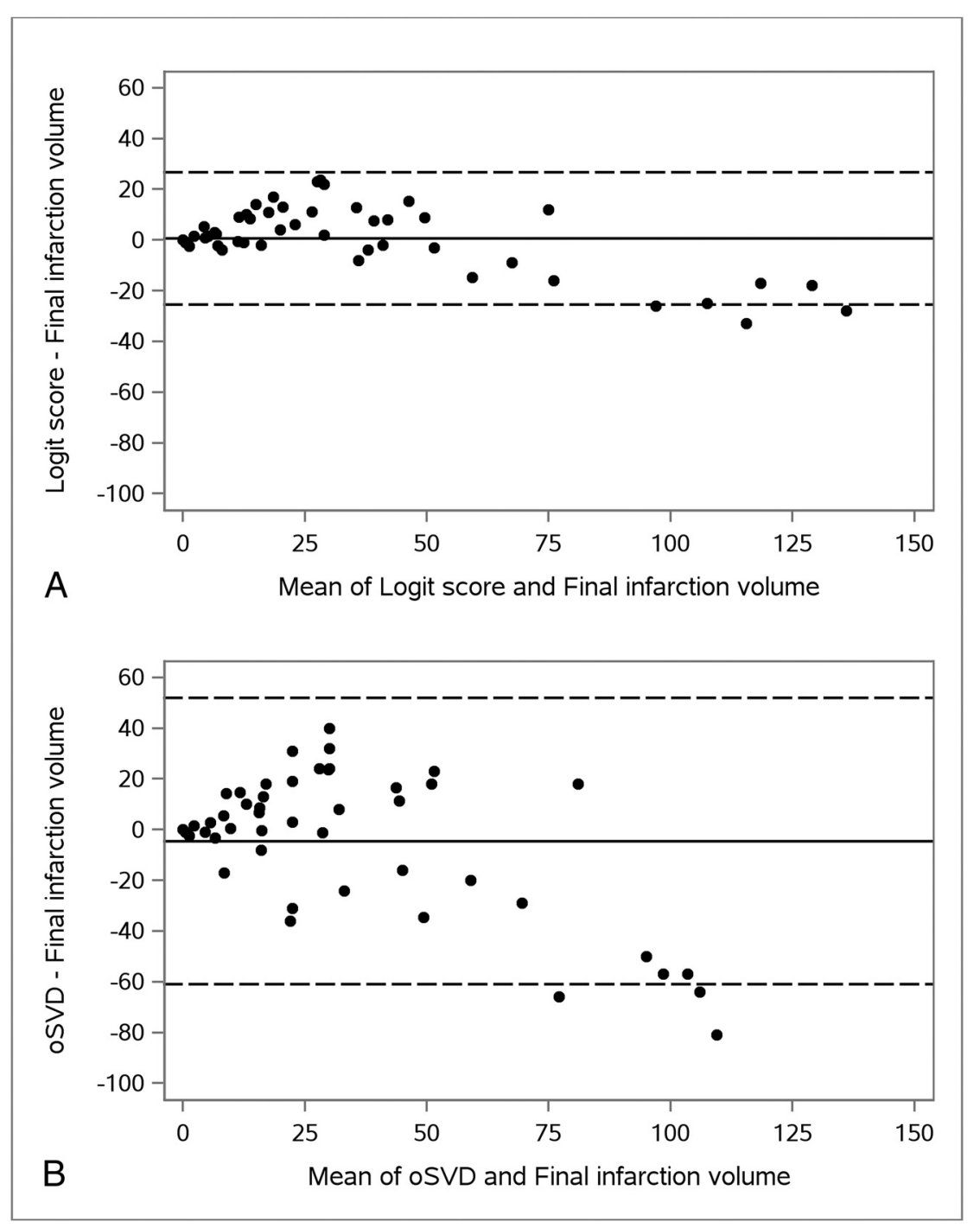

FIG 4. Bland-Altman plots of calculated final infarct volume and estimated ischemic core volume using the Bayesian-based logit score $(A)$ and oSVD-rCBF $<30 \%$ (B). Solid lines represent the mean differences. Dashed lines indicate 2 SDs above and below the mean differences. The limits of agreement were -25 to 27 for the Bayesian-based logit score and -61 to 52 for OSVD-CBF $<30 \%$.
There are several barriers to effective implementation of CTP in acute stroke care, including differences in CT scanners and hardware, postprocessing methodology such as different software packages, and different deconvolution techniques for quantitative analysis. ${ }^{30-33}$ One of the potential limitations of quantitative CTP is related to the inherently noisy nature of CTP data, which can affect postprocessing and result in erroneous calculation of the ischemic core. This limitation can become a larger problem when postprocessing is performed by deconvolution via singular value decomposition, a highly noisesensitive technique, ${ }^{34,35}$ currently used by most commercially available postprocessing software.

The theoretic advantages of the Bayesian method for more accurate estimation of perfusion parameters have been shown in digital phantom and simulation studies. ${ }^{22-24}$ A recent study in patients with AIS also showed reduced variability in CTP-estimated ischemic core volume by the Bayesian method in comparison with singular value decomposition. ${ }^{25}$

This study, to our knowledge, is the first to establish optimal thresholds for estimation of ischemic core volume using the Bayesian method. We showed that the most accurate single parameter for estimation of infarction was $\mathrm{ATD}_{\text {diff }}$ with an overall accuracy of $78.9 \%$ $(\mathrm{AUC}=0.80)$. For every 1 -second-delay difference within the ischemic territory and contralateral hemisphere, the odds ratio of a voxel being infarcted was 1.59 based approach and the currently used clinical method (oSVDrCBF $<30 \%$ ) are shown in Fig 2.

Figure 3 shows an example of CTP-estimated ischemic core using oSVD-rCBF and our Bayesian-based logit score in comparison with MR imaging.

Bland-Altman plots for comparison of CTP-estimated ischemic core volume and final infarct volume for each postprocessing method are summarized in Fig 4. The limits of agreement defined as the mean \pm 2 times the SD of the differences were -25 to 27 for the Bayesian-based logit score and -61 to 52 for oSVD-CBF.

\section{DISCUSSION}

Although quantitative CTP has been used successfully for treatment selection of patients with AIS in a controlled environment of randomized clinical trials, its day-to-day use may still be challenging due to inconsistencies of the results that are reflected in the literature. $^{13,16,17,19,25,28,29}$
(95\% CI, 1.35-1.88, $P<.001$ ). Most interesting, the threshold for Bayesian-estimated rCBF was lower than what is used currently for singular value decomposition-based methodology $(22.1 \%$ versus 30\%). This finding confirms the results of a recent study showing that Bayesian-estimated $\mathrm{rCBF}$ at the threshold of $30 \%$ can result in overestimation of the ischemic core. ${ }^{25}$ The final multiparametric Bayesian-based model provided the highest diagnostic accuracy in predicting infarction, with an overall accuracy of $79.4 \%(\mathrm{AUC}=0.84)$. In this model, if the logit score of a voxel was greater than the optimal threshold of 0.109 , the voxel would be classified as an infarct.

One advantage of our multiparametric model is that it uses a combination of time maps in addition to currently used rCBF to provide a more accurate estimation of ischemic core, in particular for patients in whom some degree of reperfusion of the ischemic core is present. Increased blood flow within the ischemic territory is a known phenomenon that increases progressively with time 
from onset, ${ }^{36-38}$ with the reported incidence of $16 \%$ at 8 hours from the onset of ischemia. ${ }^{36}$ In the absence of thrombolytic treatment, the restoration of blood flow within the ischemic core might be from reperfusion related to spontaneous recanalization of the occluded artery or via development of collateral flow or from severe reperfusion/luxury perfusion related to blood-brain barrier injury. ${ }^{39-41}$ Regardless of the underlying mechanism, following reperfusion, blood flow within the ischemic core may return to levels beyond the defined threshold (ie, $\mathrm{rCBF}<30 \%$ ), which, in turn, can result in erroneous estimation of the ischemic core volume.

The erroneous estimation of ischemic core due to infarct reperfusion can be best addressed using a combination of parametric maps, including time maps and CBF to provide supplementary information that can best approximate the physiologic status of a given voxel within the ischemic bed. Because more patients with AIS are being treated later during their disease course (up to 24 hours) and because the incidence of spontaneous reperfusion of the infarct increases with time, ${ }^{36}$ multiparametric models such as ours can provide a more realistic estimation of ischemic core volume over what is estimated through $\mathrm{rCBF}$ alone.

Our multiparametric Bayesian-based approach showed great reduction in the variability of the estimated ischemic core. Compared with the calculated final infarct volume, the limits of agreement ranged from -25 to $+27 \mathrm{~mL}$ for our multiparametric Bayesian-based model compared with -61 to $+52 \mathrm{~mL}$ for oSVDrCBF. With reliance on ischemic core volume to include or exclude patients from attempted revascularization treatment, ${ }^{14,15}$ accurate and reliable quantification of ischemic core is of paramount importance outside the controlled and supervised environment of clinical trials if CTP is to be adopted broadly in routine clinical practice. The reduced variability in the estimation of the ischemic core afforded by our multiparametric Bayesian model can play a critical role for the broad acceptance of CTP in clinical practice and to ensure that patient selection for mechanical thrombectomy is optimized.

One of the limitations of our study is that due to the retrospective study design, we did not strictly control the time between CTP and recanalization or the time between CTP and MR imaging acquisitions. Growth of infarction and increased volume are possible between CTP and the scans that were used for final infarct volume calculation. We tried to minimize these confounding factors by including patients with successful recanalization and by excluding patients with time from CTP to recanalization of $>4$ hours. Another limitation is that we used recanalization of $\geq$ TICI $2 \mathrm{~b}$ as a cutoff for successful recanalization; however, going forward and with advances in mechanical thrombectomy, $\geq$ TICI $2 c$ may be used as a cutoff for successful outcome. Last, the final imaging study used for determination of final infarct volume in our volumetric analysis was CT in a subset of patients (16\%) because they could not undergo MR imaging. Although follow-up CT has been used as an acceptable method for determination of final infarct volume, it is plausible that this can result in inconsistencies in comparison with the more accurate MR imaging.

\section{CONCLUSIONS}

We established thresholds for the Bayesian model to estimate ischemic core using CTP. The described multiparametric Bayesian-based model improved consistency in the CTP estimation of ischemic core in comparison with the methodology used in current clinical routine. If its potential is realized in a prospective study, the described model can be used for accurate estimation and reduced variability of CTP-estimated ischemic core for broader acceptance of CTP outside the controlled environment of clinical trials.

Disclosures: Kambiz Nael—UNRELATED: Board Membership: Olea Medical, Comments: Medical Advisory Board. Helena Chang-UNRELATED: Employment: Mount Sinai. J. Mocco-UNRELATED: Consultancy: Imperative Care, Cerebrotech Medical Systems, Viseon, Endostream Medical, Rebound Therapeutics, Vastrax, Comments: consultant/ownership interest; Grants/Grants Pending: Stryker, Penumbra, Medtronic, MicroVention, Comments: research support.

\section{REFERENCES}

1. Yoo AJ, Leslie-Mazwi TM, Jovin TG. Future directions in IAT: better studies, better selection, better timing and better techniques. J Neurointerv Surg 2013;5(Suppl 1):i1-6 CrossRef Medline

2. Tarpley J, Franc D, Tansy AP, et al. Use of perfusion imaging and other imaging techniques to assess risks/benefits of acute stroke interventions. Curr Atheroscler Rep 2013;15:336 CrossRef Medline

3. Ribo M, Tomasello A, Lemus M, et al. Maximal admission core lesion compatible with favorable outcome in acute stroke patients undergoing endovascular procedures. Stroke 2015;46:2849-52 CrossRef Medline

4. Jauch EC, Saver JL, Adams HP Jr, et al; American Heart Association Stroke Council, Council on Cardiovascular Nursing, Council on Peripheral Vascular Disease, Council on Clinical Cardiology. Guidelines for the early management of patients with acute ischemic stroke: a guideline for healthcare professionals from the American Heart Association/American Stroke Association. Stroke 2013;44: 870-947 CrossRef Medline

5. Schellinger PD, Bryan RN, Caplan LR, et al; Therapeutics and Technology Assessment Subcommittee of the American Academy of Neurology. Evidence-based guideline: the role of diffusion and perfusion MRI for the diagnosis of acute ischemic stroke: report of the Therapeutics and Technology Assessment Subcommittee of the American Academy of Neurology. Neurology 2010;75:177-85 CrossRef Medline

6. Kamalian S, Kamalian S, Maas MB, et al. CT cerebral blood flow maps optimally correlate with admission diffusion-weighted imaging in acute stroke but thresholds vary by postprocessing platform. Stroke 2011;42:1923-28 CrossRef Medline

7. Campbell BC, Christensen S, Levi CR, et al. Cerebral blood flow is the optimal CT perfusion parameter for assessing infarct core. Stroke 2011;42:3435-40 CrossRef Medline

8. Wintermark M, Flanders AE, Velthuis B, et al. Perfusion-CT assessment of infarct core and penumbra: receiver operating characteristic curve analysis in $\mathbf{1 3 0}$ patients suspected of acute hemispheric stroke. Stroke 2006;37:979-85 Medline

9. Nogueira RG, Jadhav AP, Haussen DC, et al; DAWN Trial Investigators. Thrombectomy 6 to 24 hours after stroke with a mismatch between deficit and infarct. N Engl J Med 2018;378:11-21 CrossRef Medline

10. Albers GW, Marks MP, Kemp S, et al; DEFUSE 3 Investigators. Thrombectomy for stroke at 6 to 16 hours with selection by perfusion imaging. N Engl J Med 2018;378:708-18 CrossRef Medline

11. Powers WJ, Rabinstein AA, Ackerson T, et al; American Heart Association Stroke Council. 2018 Guidelines for the Early Management of Patients with Acute Ischemic Stroke: a Guideline for Healthcare Professionals from the American Heart Association/American Stroke Association. Stroke 2018;49:e46-110 CrossRef Medline 
12. González RG. Current state of acute stroke imaging. Stroke 2013;44: 3260-64 CrossRef Medline

13. Schaefer PW, Souza L, Kamalian S, et al. Limited reliability of computed tomographic perfusion acute infarct volume measurements compared with diffusion-weighted imaging in anterior circulation stroke. Stroke 2015;46:419-24 CrossRef Medline

14. Saver JL, Goyal M, Bonafe A, et al; SWIFT PRIME Investigators. Stent-retriever thrombectomy after intravenous t-PA vs. t-PA alone in stroke. $N$ Engl J Med 2015;372:2285-95 CrossRef Medline

15. Campbell BC, Mitchell PJ, Kleinig TJ, et al; EXTEND-IA Investigators. Endovascular therapy for ischemic stroke with perfusion-imaging selection. N Engl J Med 2015;372:1009-18 CrossRef Medline

16. Copen WA, Morais LT, Wu O, et al. In acute stroke, can CT perfusion-derived cerebral blood volume maps substitute for diffusionweighted imaging in identifying the ischemic core? PLoS One 2015; 10:e0133566 CrossRef Medline

17. Bandera E, Botteri M, Minelli C, et al. Cerebral blood flow threshold of ischemic penumbra and infarct core in acute ischemic stroke: a systematic review. Stroke 2006;37:1334-39 Medline

18. Turowski B, Schramm P. An appeal to standardize CT- and MR-perfusion. Clin Neuroradiol 2015;25(Suppl 2):205-10 CrossRef Medline

19. Huisa BN, Neil WP, Schrader R, et al. Clinical use of computed tomographic perfusion for the diagnosis and prediction of lesion growth in acute ischemic stroke. J Stroke Cerebrovasc Dis 2014;23: 114-22 CrossRef Medline

20. Geuskens RR, Borst J, Lucas M, et al; MR CLEAN trial investigators (www.mrclean-trial.org). Characteristics of misclassified CT perfusion ischemic core in patients with acute ischemic stroke. PLoS One 2015;10:e0141571 CrossRef Medline

21. Mouridsen K, Friston K, Hjort N, et al. Bayesian estimation of cerebral perfusion using a physiological model of microvasculature. Neuroimage 2006;33:570-79 Medline

22. Boutelier T, Kudo K, Pautot F, et al. Bayesian hemodynamic parameter estimation by bolus tracking perfusion weighted imaging. IEEE Trans Med Imaging 2012;31:1381-95 CrossRef Medline

23. Sasaki M, Kudo K, Boutelier T, et al. Assessment of the accuracy of a Bayesian estimation algorithm for perfusion CT by using a digital phantom. Neuroradiology 2013;55:1197-203 CrossRef Medline

24. Kudo K, Boutelier T, Pautot F, et al. Bayesian analysis of perfusionweighted imaging to predict infarct volume: comparison with singular value decomposition. Magn Reson Med Sci 2014;13:45-50 CrossRef Medline

25. Sakai Y, Delman BN, Fifi JT, et al. Estimation of ischemic core volume using computed tomographic perfusion. Stroke 2018;49: 2345-52 CrossRef Medline

26. Mouridsen K, Christensen S, Gyldensted L, et al. Automatic selection of arterial input function using cluster analysis. Magn Reson Med 2006;55:524-31 Medline

27. Wu O, Østergaard L, Weisskoff RM, et al. Tracer arrival timing-insensitive technique for estimating flow in MR perfusion-weighted imag- ing using singular value decomposition with a block-circulant deconvolution matrix. Magn Reson Med 2003;50:164-74 Medline

28. Hasan TF, Rabinstein AA, Middlebrooks EH, et al. Diagnosis and management of acute ischemic stroke. Mayo Clin Proc 2018;93: 523-38 CrossRef Medline

29. Xin Y, Han FG. Diagnostic accuracy of computed tomography perfusion in patients with acute stroke: a meta-analysis. J Neurol Sci 2016;360:125-30 CrossRef Medline

30. Konstas AA, Goldmakher GV, Lee TY, et al. Theoretic basis and technical implementations of CT perfusion in acute ischemic stroke, Part 1: theoretic basis. AJNR Am J Neuroradiol 2009;30:662-68 CrossRef Medline

31. Bivard A, Levi C, Spratt N, et al. Perfusion CT in acute stroke: a comprehensive analysis of infarct and penumbra. Radiology 2013; 267:543-50 CrossRef Medline

32. Goyal M, Menon BK, Derdeyn CP. Perfusion imaging in acute ischemic stroke: let us improve the science before changing clinical practice. Radiology 2013;266:16-21 CrossRef Medline

33. Kudo K, Sasaki M, Yamada K, et al. Differences in CT perfusion maps generated by different commercial software: quantitative analysis by using identical source data of acute stroke patients. $R a-$ diology 2010;254:200-09 CrossRef Medline

34. Konstas AA, Goldmakher GV, Lee TY, et al. Theoretic basis and technical implementations of CT perfusion in acute ischemic stroke, Part 2: technical implementations. AJNR Am J Neuroradiol 2009;30: 885-92 CrossRef Medline

35. Fieselmann A, Kowarschik M, Ganguly A, et al. Deconvolution based $C T$ and MR brain perfusion measurement: theoretical model revisited and practical implementation details. Int J Biomed Imaging 2011:2011:467563 CrossRef Medline

36. Rubin G, Firlik AD, Levy EI, et al. Xenon-enhanced computed tomography cerebral blood flow measurements in acute cerebral ischemia: review of 56 cases. J Stroke Cerebrovasc Dis 1999;8:404-11 Medline

37. Hakim AM, Pokrupa RP, Villanueva J, et al. The effect of spontaneous reperfusion on metabolic function in early human cerebral infarcts. Ann Neurol 1987;21:279-89 Medline

38. Jørgensen HS, Sperling B, Nakayama H, et al. Spontaneous reperfusion of cerebral infarcts in patients with acute stroke: incidence, time course, and clinical outcome in the Copenhagen Stroke Study. Arch Neurol 1994;51:865-73 Medline

39. Shimosegawa E, Hatazawa J, Inugami A, et al. Cerebral infarction within six hours of onset: prediction of completed infarction with technetium-99m-HMPAO SPECT. J Nucl Med 1994;35:1097-103 Medline

40. Baron JC, Bousser MG, Comar D, et al. Noninvasive tomographic study of cerebral blood flow and oxygen metabolism in vivo: potentials, limitations, and clinical applications in cerebral ischemic disorders. Eur Neurol 1981;20:273-84 Medline

41. Lin L, Bivard A, Parsons MW. Perfusion patterns of ischemic stroke on computed tomography perfusion. J Stroke 2013;15:164-73 CrossRef Medline 